\title{
QUALIDADE DE VIDA DA PESSOA IDOSA CONFORME NIVEL DE INSTITUCIONALIZAÇÃO
}

Andrea Mendes Araújo ${ }^{1}$

Ângelo José Gonçalves Bós²

resumo

Introdução: Pesquisas apontam efeito negativo da institucionalização da pessoa idosa para a sua qualidade de vida. Objetivo: Avaliar possíveis diferenças na qualidade de vida da pessoa idosa em lista de espera para residir em instituição de longa permanência (espera), residente em instituição (residente), e daquele que não está em lista de espera (não espera). Método: Estudo transversal. Entrevistados 50 componentes de cada grupo, pareados por sexo, idade e escolaridade. Utilizados os instrumentos WHOQOL-BREF e WHOQOL-OLD. Resultados: Encontrou-se diferença significativa em todos os domínios e questões do WHOQOL-BREF entre os três grupos, com médias do escore total de: 62,0 $\pm 10,61,44,1 \pm 13,63$ e 68,8 $\pm 7,07$, respectivamente, para residente, espera e não espera. Importantes diferenças também foram identificadas na avaliação do WHOQOL-

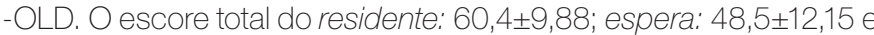

1 Graduada em Enfermagem. Doutora em Gerontologia Biomédica. Professora da Escola Técnica de Saúde da Universidade Federal da Paraíba (ETS/UFPB). E-mail: andreamendesetspb@hotmail.com. 2 Graduado em Medicina. pós-doutorado no Instituto Nacional Americano sobre o Envelhecimento e na Escola de Saúde Pública da Universidade de Johns Hopkins em Baltimore, EUA, Professor Adjunto da Pontifícia Universidade Católica do Rio Grande do Sul (PUCRS), Brasil. E-mail: angelo.bos@pucrs.br. 


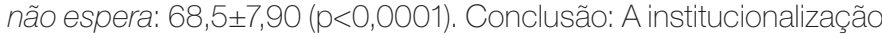
não proporciona piora na qualidade de vida da pessoa idosa, a percepção dessa qualidade de vida pode já estar comprometida quando se procura a institucionalização.

palavras-chave

Envelhecimento. Idoso. Qualidade de Vida. Listas de espera.

\section{Introdução}

Os índices crescentes de pessoas idosas impõem situações desafiadoras ao poder público, sociedade, família e indivíduos, no que se refere à elaboração de estratégias que favoreçam uma longevidade com preservação da autonomia e independência, alicerçada na promoção de uma vida ativa e saudável (LITIVOC; BRITO, 2004). O Ministério da Saúde estabelece que na atenção à pessoa idosa devam ser enfatizadas as iniciativas dirigidas à proteção, manutenção e recuperação de sua capacidade funcional, incluindo medidas que visem à promoção da qualidade de vida, do envelhecimento ativo e a proximidade com a família e a comunidade o maior tempo possível (BRASIL, 2006).

O termo qualidade de vida expressa a maneira como o indivíduo está adaptado às atividades do seu cotidiano, incluindo seu estado de saúde que envolve bem estar físico mental, funcional e sua inclusão social (NERI, 2008). Destaca-se a percepção que o indivíduo tem do espaço que ocupa na vida dentro do contexto social, cultural e do sistema de valores de onde vive, e em relação a seus objetivos, expectativas, padrões e preocupações (FLECK, 2008).

O processo de transição demográfica vem proporcionando alterações consideráveis no perfil de saúde da pessoa idosa, convivência maior com doenças crônicas e incapacidade funcional, favorecendo a uma maior vulnerabilidade e dependência, aumentando os demandantes de cuidados em meio à diminuição de descendentes para cuidar. A família, que culturalmente é a provedora de cuidados, sofre transformações estruturais decorrentes da queda da natalidade, mudanças nos padrões de nupcialidade e o ingresso da mulher no mercado de trabalho (CAMARANO; KANSO, 2010a).

Entre as alternativas para o cuidado não familiar da pessoa idosa, as mais antigas e conhecidas são as instituições asilares, que no Brasil foram renomeadas pela Sociedade Brasileira de Geriatria e Gerontologia (SBGG), para Instituição de Longa Permanência para Idosos (ILPI). Essa modalidade de cuidado sempre 
foi vista com resistência e preconceito, assemelhada a "depósitos de pessoas idosas" e à finitude da vida (CAMARANO; SCHARFSTEIN, 2010).

As ILPIs surgem para suprir a necessidade de guarda, proteção, segurança e alimentação, abrigando as pessoas idosas rejeitadas pelos seus grupos diretos, à medida que sua presença torna-se incômoda, difícil e insustentável, ficando sua participação familiar e social limitada ou até impossibilitada (CAMARANO; SCHARFSTEIN, 2010). A instituição oferece condições melhores do que viver nas ruas ou serem vítimas de maus tratos familiares. Favorece a construção e manutenção de vínculos afetivos e não reduz a importância da família no apoio e cuidado de seus membros (CHRISTOPHE; CAMARANO, 2010). Significa uma nova organização e divisão de responsabilidade entre os "pilares do bem estar" (CAMARANO; PASINATO, 2004), família, estado e mercado (CHRISTOPHE; CAMARANO, 2010).

Poucos são os estudos (OLIVEIRA; NOVAES, 2013; QUEIROZ, 2010; DANILOW et al., 2007) que evidenciam dados positivos da institucionalização; a maioria demonstra (OLIVEIRA; GOMES; PAIVA, 2011; FREITAS; SCHEICHER, 2010; SAGALLA et al., 2013; RAMOS, 2008; SANTOS et al., 2017) que a qualidade de vida da pessoa idosa institucionalizada é inferior ao da não institucionalizada, que vive na comunidade e que provavelmente não tenha a necessidade de residir numa ILPI, reforçando negativamente o conceito que se tem destas instituições. Contesta-se nesses estudos a escolha do grupo de comparação. Será que a qualidade de vida da pessoa idosa residente em ILPI, se comparada com aquela que apresenta necessidade de apoio à moradia ou cuidados com a saúde, teria os mesmos resultados?

Solicitar vaga para residir em ILPI é uma decisão que acarreta sofrimento e geralmente só é tomada quando a pessoa idosa apresenta incapacidades funcionais. Aqueles que as solicitam, antes da instalação de comprometimentos físicos e mentais, também são atormentados pelas imagens negativas que acompanham esses serviços. Pesquisas na área mostram dados que potencializam essa concepção. Comparam a qualidade de vida da pessoa idosa residente em ILPI com a residente na comunidade. Estatisticamente provam que pessoa idosa na comunidade vive com mais qualidade (OLIVEIRA; GOMES; PAIVA, 2011; RAMOS, 2008).

No processo de envelhecimento destaca-se o aumento da demanda por ILPI, considerada uma alternativa de moradia (CAMARANO; SCHARFSTEIN, 2010; POLLO; ASSIS, 2008; POLARO et al., 2012), e assistência à saúde (MINICATO; FREITAS, 2007). A cobertura dessa modalidade de atendimento é baixa, talvez reflexo da imagem pejorativa que acompanha a institucionalização (CAMARANO; KANSO, 2010a; CAMARANO; KANSO, 2010b). Tem-se 
a concepção que a pessoa idosa só reside em ILPI após ter esgotado todas as outras possibilidades de moradia, e esta demanda sofrimento à pessoa idosa. Os estudos sobre institucionalização das pessoas idosas deixam no anonimato dados referentes a uma parcela considerável dessa população que aguarda vaga em lista de espera para residir em ILPI. Existem pontuais citações da sua existência (POLLO; ASSIS, 2008; POLARO et al., 2012), mas não foi encontrada, na literatura brasileira, a descrição dessa amostra. Considerando as peculiaridades da pessoa idosa institucionalizada e a carência de estudos que abordem a parcela populacional em lista de espera, surgiu a necessidade de se avaliar e comparar a qualidade de vida das pessoas idosas residentes em ILPI (residente), daquelas residentes na comunidade que não demonstrem interesse em residir em ILPI (não espera) e das que se encontram em lista de espera para residir em ILPI (espera).

Diante do exposto, justifica-se o propósito desta pesquisa, uma vez que existe a necessidade de discutir e formular novos conceitos sobre a institucionalização da pessoa idosa no Brasil. Pretende-se apresentar à sociedade e às autoridades públicas, dados provenientes de uma abordagem diferenciada dessa parcela da população idosa, com tendências a um crescimento vertiginoso.

\section{Metodologia}

Estudo transversal, realizado com pessoas idosas (idade $\geq 60$ anos) na cidade de João Pessoa/PB. Consideraram-se neste estudo níveis de institucionalização caracterizados em três grupos: pessoa idosa residente em ILPI, identificada como residente; que aguarda vaga em lista de espera para residir em ILPI, referida nesse trabalho como espera; e a pessoa idosa que não está em lista de espera e não demonstra interesse em residir em ILPI, nomeada grupo não espera.

A população idosa que aguardava vaga em ILPI foi encontrada, utilizando-se a lista de espera obtida nas ILPIs da cidade de João Pessoa. Foram consideradas todas as pessoas idosas que solicitaram vaga nos anos de 2010 a 2012 nas ILPIs. Das seis ILPIs pesquisadas, uma não trabalhava com lista de espera. Segundo seu presidente "a procura era grande e quando surgia vaga logo era preenchida”. Duas outras possuíam apenas alguns nomes registrados, pois até então não haviam pensado na importância de se manter uma lista de espera atualizada, sendo que uma delas havia descartado parte dos registros dos anos 2011 e 2012. Foram localizadas 690 solicitações de vaga, destas foi realizado contato telefônico com 409 registros (70 pessoas idosas solicitaram vaga em mais de uma instituição) e 58 autorizações para visita domiciliar, 
sendo que 08 pessoas idosas não possuíam funções cognitivas preservadas para responder o questionário da pesquisa.

O contato com as pessoas idosas do grupo espera foi realizado pela pesquisadora e colaboradores, previamente treinados. Na oportunidade se explicava por telefone o objetivo do contato e na ocorrência de cognição preservada do pretendente a vaga, solicitava-se autorização para visita domiciliar ao responsável e/ou à própria pessoa idosa. Foram observadas várias dificuldades para acesso à pessoa idosa, entre elas citam-se: telefones desligados ou inexistentes, números desconhecidos, familiares que recusaram falar no assunto, pessoas idosas e/ou familiares que recusaram receber visita, pessoas idosas em óbito, familiares que negaram a solicitação da vaga.

O recrutamento do grupo não espera foi concomitante ao grupo espera. Para cada respondente desse grupo se localizava na mesma rua ou bairro, o respondente do grupo não espera e deveria apresentar características semelhantes ao espera, conforme critério de pareamento. A princípio era solicitado ao respondente do grupo espera indicação de outra pessoa idosa na mesma rua ou bairro. Na impossibilidade de localizar com as informações do respondente, os entrevistadores procuravam através da sondagem nas residências. Não foi solicitado apoio às igrejas, associação de bairro ou qualquer outra entidade, para evitar viés na amostra.

Quanto ao grupo residente em ILPI, para cada 10 entrevistas na comunidade ( 5 residente +5 espera), realizavam-se 5 entrevistas na ILPI para a qual o respondente do grupo espera solicitou a vaga. As pessoas idosas residentes foram localizadas através dos registros das instituições.

Para o alcance dos propósitos desta pesquisa adotaram-se estratégias que pudessem expressar a realidade dos grupos em estudo e minimizar a ocorrência de vieses. Assim sendo, foram admitidos os seguintes critérios de pareamento: possuir o mesmo sexo; mesma idade ou diferença máxima de 03 anos; mesma escolaridade ou variação de 01 ano de estudo, tendo como referência o grupo espera.

Na coleta de dados, que ocorreu entre os meses de maio de 2012 e fevereiro de 2013, utilizou-se o Mini Exame do Estado Mental (FOLSTEIN, M.; FOLSTEIN, S.; MCHUGH, 1975) para avaliar a função cognitiva (atendendo ao critério de exclusão, foram considerados ponto de corte 18, para as pessoas idosas não alfabetizadas, e 24, para aquelas com um ou mais anos de estudo) e na avaliação da qualidade de vida os questionários: WHOQOL-BREF (refere-se a uma versão abreviada do instrumento genérico WHOQOL 100, engloba 26 questões distribuídas em quatro domínios (físico, psicológico, relações sociais e meio ambiente) e duas questões gerais sobre qualidade de vida) e WHOQOL-OLD 
(instrumento específico para avaliar a qualidade de vida das pessoas idosas; contém 24 itens, com resposta por escala tipo Likert de 1 a 5, divididos em seis facetas: Funcionamento do Sensório (FS), Autonomia (AUT), Morte e Morrer (MEM), Atividades Passadas, Presentes e Futuras (APPF), Participação Social (PSO) e Intimidade (INT). Os dados foram organizados em um banco de dados no programa EPI INFO versão 3.5.3, onde foram analisados através da estatística descritiva e analítica, com os resultados distribuídos em tabelas.

A média dos escores de cada componente do questionário WHOQOL-BREF e WHOQOL-OLD foi comparada entre os grupos pela ANOVA. Quando a ANOVA foi significativa, dois testes t de Student foram realizados entre o grupo residente e o grupo espera e este com o grupo não espera. Os testes que obtiveram um $\mathrm{p}<0,05$ foram considerados como significativos (um erro tipo alfa de $5 \%$ ).

A pesquisa seguiu as diretrizes do Conselho Nacional de Ética em Pesquisa. Foi apresentado ao Conselho Municipal do Idoso do município de João Pessoa para autorização da conselheira presidente. Posteriormente, foi dirigido a cada representante legal das ILPIs, para a obtenção das cartas de anuências e só então submetido ao Comitê de Ética em Pesquisa da UFPB, aprovado em 2 de maio de 2012, parecer n⿳0 17123 .

\section{Resultados}

Fizeram parte desse estudo 150 pessoas idosas igualmente distribuídas segundo nível de institucionalização em três grupos: residente (50), espera (50) e não espera (50). Sendo $76 \%(p=1,0000)$ do sexo feminino nos três grupos; com média de idade entre os grupos variando no máximo em 2,5 anos $(p=0,2166)$ e os anos de estudo variando entre 6,3 no residente e 7,2 no não espera $(p=0,7002)$. Não houve diferença estatística entre os grupos para essas variáveis, haja vista que foram utilizados critérios de pareamento na seleção da amostra.

A Tabela 1 apresenta a distribuição das questões gerais e dos domínios do instrumento WHOQOL-BREF de acordo com o grupo, encontra-se descrita no final do artigo, apêndice A. Os escores produzidos nas questões gerais, autopercepção da qualidade de vida $(p<0,0001)$ e satisfação com a saúde $(p<0,0001)$, demonstram diferença estatisticamente significativa para os grupos residente, espera e não espera, sendo as maiores médias no grupo não espera e as menores no grupo espera, assim como na comparação entre os grupos todos foram estatisticamente diferentes entre eles - espera/residente $(p=0,0003 ; p=0,0239)$ e espera/não espera $(\mathrm{p}<0,0001 ; \mathrm{p}<0,0001)$, respectivamente, para as questões autopercepção da qualidade de vida e satisfação com a saúde. 
Tabela 1 - Distribuição das questões gerais e dos domínios do instrumento WHOQOL-BREF de acordo com o grupo de pessoas idosas, João Pessoa-PB, maio de 2012 a fevereiro de 2013.

\begin{tabular}{|c|c|c|c|c|c|c|c|}
\hline & \multicolumn{3}{|c|}{ GRUPO } & \multicolumn{4}{|c|}{$p$} \\
\hline & $\begin{array}{l}\text { Residente } \\
\quad(n=50)\end{array}$ & $\begin{array}{l}\text { Espera } \\
(n=50)\end{array}$ & $\begin{array}{l}\text { Não } \\
\text { espera } \\
(n=50)\end{array}$ & Todos & $\begin{array}{c}\text { Espera } \\
\text { resi- } \\
\text { dente/ }\end{array}$ & $\begin{array}{l}\text { Espera/ } \\
\text { Não } \\
\text { espera }\end{array}$ & $\begin{array}{c}\text { Resi- } \\
\text { dente/ } \\
\text { Não } \\
\text { espera }\end{array}$ \\
\hline \multicolumn{8}{|l|}{ Questões } \\
\hline Aut_QV & $3,6 \pm 0,78$ & $2,9 \pm 1,10$ & $4,0 \pm 0,60$ & $<0,0001^{*}$ & $0,0003^{*}$ & $<0,0001^{*}$ & 0,0236 \\
\hline $\begin{array}{l}\text { satis_- } \\
\text { saúde }\end{array}$ & $3,3 \pm 0,88$ & $2,9 \pm 0,94$ & $3,8 \pm 0,59$ & $<0,0001^{\star}$ & 0,0239 & $<0,0001^{*}$ & $0,0034^{*}$ \\
\hline \multicolumn{8}{|l|}{ Domínios } \\
\hline Físico & $59,7 \pm 16,28$ & $44,1 \pm 19,65$ & $71,0 \pm 13,95$ & $<0,0001$ & $<0,0001$ & $<0,0001^{*}$ & 0,0003 \\
\hline $\begin{array}{l}\text { Psicoló- } \\
\text { gico }\end{array}$ & $64,3 \pm 15,50$ & $44,1 \pm 18,96$ & $71,0 \pm 9,99$ & $<0,0001$ & $<0,0001$ & $<0,0001^{*}$ & $0,0121^{*}$ \\
\hline $\begin{array}{l}\text { Relações } \\
\text { sociais }\end{array}$ & $64,6 \pm 13,32$ & $46,8 \pm 16,91$ & $74,0 \pm 11,50$ & $<0,0001$ & $<0,0001$ & $<0,0001^{*}$ & 0,0003 \\
\hline $\begin{array}{l}\text { Meio } \\
\text { ambiente }\end{array}$ & $61,3 \pm 9,59$ & $43,1 \pm 12,98$ & $63,2 \pm 10,03$ & $<0,0001$ & $<0,0001$ & $<0,0001$ & 0,3261 \\
\hline $\begin{array}{l}\text { Escore } \\
\text { Total }\end{array}$ & $62,0 \pm 10,61$ & $44,1 \pm 13,63$ & $68,8 \pm 7,02$ & $<0,0001$ & $<0,0001$ & $<0,0001^{*}$ & 0,0014 \\
\hline
\end{tabular}

Aut_QV - Autopercepção da qualidade de vida; Satis_saúde - Satisfação com a saúde. *Teste Kruskal-Wallis

Fonte: Tabela elaborada pelos autores.

Em relação aos domínios do questionário WHOQOL-BREF, houve diferença estatisticamente significativa em todos os domínios com $\mathrm{p}<0,0001$, em relação aos três grupos. Ao comparar os grupos em espera/residente, espera/não espera e residente/não espera, as significâncias mantiveram-se, exceto para o domínio meio ambiente em relação aos grupos residente/não espera $(\mathrm{p}=0,3261)$, conforme Tabela 1.

O escore total do WHOQOL-BREF foi de $62,0 \pm 10,61$ para o grupo residente, $44,1 \pm 13,63$ para o grupo espera e de $68,8 \pm 7,02$ para o grupo não espera, evidenciando mais uma vez a diferença estatística que existe entre os três grupos estudados $(\mathrm{p}<0,0001)$, assim como, mantém uma diferença significativa na seleção de dois grupos espera/residente, espera/não espera $(\mathrm{p}<0,0001)$, residente/não espera $(\mathrm{p}<0,0001)$ e residente/não espera $(\mathrm{p}=0,0014)$ - ver Tabela 1 . Em todos os escores do WHOQOL-BREF o grupo espera foi estatisticamente menor que os outros dois grupos, enquanto o grupo não espera obteve sempre os melhores escores. 
A Tabela 2 mostra a distribuição das facetas do instrumento WHOQOL-OLD e grupo pesquisado, encontra-se exposta no final do artigo, apêndice B. O grupo espera apresentou invariavelmente os piores escores atribuídos às facetas. A única faceta que não houve diferença estatística foi a Morte e Morrer $-\operatorname{MEM}(\mathrm{p}=0,8007)$. Ao comparar os grupos em pares também se constatou significância estatística, exceto para MEM nas três comparações e para a faceta Funcionamento do Sensório - FS, $(\mathrm{p}=0,1889)$ entre os grupos residente e não espera.

Tabela 2 - Distribuição das facetas do instrumento do WHOQOL-OLD de acordo com o grupo de pessoas idosas, João Pessoa-PB, maio de 2012 a fevereiro de 2013.

\begin{tabular}{|c|c|c|c|c|c|c|c|}
\hline \multirow[b]{2}{*}{ Facetas } & \multicolumn{3}{|c|}{ GRUPO } & \multicolumn{4}{|c|}{$p$} \\
\hline & $\begin{array}{l}\text { Residente } \\
\quad(n=50)\end{array}$ & $\begin{array}{l}\text { Espera } \\
(n=50)\end{array}$ & $\begin{array}{l}\text { Não } \\
\text { espera } \\
(n=50)\end{array}$ & Todos & $\begin{array}{c}\text { Espera/ } \\
\text { resi- } \\
\text { dente }\end{array}$ & $\begin{array}{c}\text { Espera/ } \\
\text { Não } \\
\text { espera }\end{array}$ & $\begin{array}{l}\text { Resi- } \\
\text { dente/ } \\
\text { Não } \\
\text { espera }\end{array}$ \\
\hline FS & $76,5 \pm 22,32$ & $62,0 \pm 27,11$ & $82,7 \pm 16,73$ & $<0,0001$ & 0,0043 & 0,0001 & $0,1889^{*}$ \\
\hline AUT & $52,1 \pm 16,83$ & $41,1 \pm 19,40$ & $64,2 \pm 12,11$ & $<0,0001$ & 0,0031 & $<0,0001^{*}$ & 0,0001 \\
\hline MEM & $71,1 \pm 23,31$ & $67,7 \pm 28,53$ & $68,9 \pm 25,14$ & 0,8007 & 0,5186 & 0,8347 & 0,6437 \\
\hline PPF & $50,5 \pm 10,55$ & $39,5 \pm 11,67$ & $57,1 \pm 10,49$ & $<0,0001$ & $<0,0001$ & $<0,0001$ & 0,0022 \\
\hline PSO & $55,4 \pm 14,45$ & $39,5 \pm 18,14$ & $66,4 \pm 10,85$ & $<0,0001$ & $<0,0001$ & $<0,0001^{*}$ & $0,0002^{*}$ \\
\hline INT & $56,6 \pm 20,65$ & $40,9 \pm 23,76$ & $71,7 \pm 11,79$ & $<0,0001$ & 0,0006 & $<0,0001^{*}$ & $0,0001^{*}$ \\
\hline $\begin{array}{l}\text { Escore } \\
\text { Total }\end{array}$ & $60,4 \pm 9,88$ & $48,5 \pm 12,15$ & $68,5 \pm 7,90$ & $<0,0001$ & $<0,0001$ & $<0,0001^{\star}$ & 0,0001 \\
\hline
\end{tabular}

FS - Funcionamento do sensório; AUT - Autonomia; MEM - Morte e Morrer; PPF Atividades presentes, passadas e futuras; PSO - participação Social; INT - Intimidade. *Teste Kruskal-Wallis

Fonte: Tabela elaborada pelos autores.

As médias produzidas no resultado do escore total dos grupos residente, espera e não espera, respectivamente, foram: 60,4 49,$88 ; 48,5 \pm 12,15$ e 68,5 $\pm 7,90$, com significância estatística entre as médias dos três grupos $(\mathrm{p}<0,0001)$. $\mathrm{O}$ valor de p também se manteve na comparação de dois grupos espera/não espera, esperal residente e residente/não espera.

\section{Discussões}

Para a avaliação da qualidade de vida da pessoa idosa, seguiram-se as orientações do manual do instrumento específico WHOQOL-OLD, que 
recomenda aplicar em conjunto com o WHOQOL-BREF (WHO, 2005a). No que se refere aos escores de cada domínio da qualidade de vida, não existe um ponto de corte que determine uma "boa" ou "ruim" qualidade de vida (PASKULIN, 2006). Segundo Fleck et al. (2000), quanto mais alto o escore, melhor a qualidade de vida.

Ao fazer a comparação entre os grupos, perceberam-se menores médias nos escores produzidos pelo grupo espera em relação aos grupos residente e este menor que o não espera. Identificaram-se diferenças significativas entre os três grupos em todos os domínios do WHOQOL-BREF e as facetas do WHOQOL -OLD, exceto na faceta morte e morrer, que faz referência às preocupações, inquietações e temores sobre a finitude (POWER, 2008). Essa faceta, além de não apresentar significância entre os grupos foi a única faceta ou domínio ma qual o não espera não foi maior que o residente.

Pesquisa realizada em São Paulo, através da Fundação Perseu Abramo (NERI, 2007), mostrou um percentual elevado de pessoas idosas (81\%) que referiram não temer a morte. As razões pontuadas na pesquisa foram: associação da velhice com a finitude, considerar um evento inevitável e não exercer domínio sobre sua ocorrência. Para Neri (2007) morrer faz parte do percurso natural da vida, sendo comum a todas as pessoas, independente da classe social ou ambiente que está inserido. A dependência como desfecho da idade mais avançada, constitui-se em um dos maiores temores desta faixa etária (BRASIL, 2007).

Ao comparar os resultados da qualidade de vida em pares de grupos constatou-se que quase todas as comparações foram significativas, demonstrando que todos os grupos são diferentes entre si em cada um dos domínios e facetas. As poucas exceções, além do enfoque morte e morrer, ocorreram entre os grupos residente/não espera, nos quais não houve diferença estatística na percepção dos respondentes desses grupos para o domínio meio ambiente e a faceta Funcionamento do Sensório, que analisa o funcionamento sensorial e o impacto das perdas das habilidades sensoriais na qualidade de vida (POWER, 2008).

Por se tratar de um estudo inédito na abordagem da qualidade de vida de pessoas idosas em lista de espera para ILPI, não se dispõe de dados de outras pesquisas para possíveis comparações. Encontra-se na literatura uma pesquisa realizada por Oliveira, Gomes e Paiva, (2011) com 70 pessoas idosas, funcionalmente independentes, residentes em ILPI, e 210 pessoas idosas não residentes, com diferenças significativas em todos os domínios da qualidade de vida avaliados pelo WHOQOL-BREF.

No estudo anteriormente referido as pessoas idosas institucionalizadas apresentaram pior qualidade de vida em comparação às pessoas idosas não institucionalizadas, (OLIVEIRA; GOMES; PAIVA, 2011) e esses resultados 
também foram observados em outras pesquisas (FREITAS; SCHEICHER, 2010; MINICATO; FREITAS, 2007; SAGALLA et al., 2013; RAMOS, 2008). O presente estudo também verificou escores piores de qualidade de vida nas pessoas idosas residentes em ILPI, quando comparadas com o grupo não espera, entretanto, as pessoas idosas em lista de espera apresentaram invariavelmente escores ainda piores do que os residentes em instituição.

Dias, Carvalho e Araújo (2013), em pesquisa realizada com pessoas idosas que vivem sozinhas, com a família e institucionalizados, não foi citado uso de pareamento, não encontraram diferenças significativas nos grupos, exceto pelo domínio físico, no qual teve melhor percepção o grupo das pessoas idosas institucionalizadas em comparação com o grupo das pessoas idosas que vivem com a família. Tal diferença estatística também foi observada nesse estudo para a avaliação do domínio físico entre os grupos, no entanto a pior média foi apresentada pelo grupo espera, e a melhor pelo grupo não espera. No resultado do escore total desses instrumentos, assim como em seus domínios e facetas, pode-se constatar que os escores produzidos pelos residentes são mais próximos das médias do grupo não espera, levando a supor que respondentes do grupo espera correspondem a uma população com características diferenciadas, apresentando níveis de qualidade de vida inferiores às outras pessoas idosas dos dois grupos estudados.

Em suma, achados dessa pesquisa mostra que a pessoa idosa quando busca ILPI apresenta comprometimentos na sua qualidade de vida. A institucionalização atua nesse contexto para mitigar os efeitos insalubres produzidos pelo contexto social e/ou familiar da pessoa idosa. A análise dos dados leva a uma hipótese muito forte que a institucionalização melhora a qualidade de vida das pessoas que sentem a necessidade desse recurso, ao contrário do que toda a literatura busca comprovar, ou seja, a institucionalização estaria relacionada à piora na qualidade de vida. Oliveira et al. (2015) comparou a qualidade de vida das pessoas idosas que vivem em ILPIs com a qualidade de vida daquelas que vivem em cuidados domiciliares, constatou classificação satisfatória da qualidade de vida das duas populações em estudo.

No tocante aos componentes da qualidade de vida que apresentam diferença entre as pessoas idosas nos grupos espera, residente e não espera, detectou-se entre o grupo espera que as médias mais baixas na avaliação da qualidade de vida foram atribuídas ao domínio meio ambiente do questionário WHOQOL-BREF, demonstrando insatisfação desta população com o meio em que vive. Para a Organização Mundial de Saúde (WHO, 2005b), o ambiente físico é importante, pois pode representar a diferença entre a dependência e independência da pessoa idosa. No Brasil existe uma carência de mudança no 
contexto atual, no sentido de produção de um ambiente social e cultural mais favorável à velhice (BRASIL, 2007). Um apoio social inadequado está associado ao aumento da mortalidade, morbidade, problemas psicológicos, diminuição da saúde e do bem-estar em geral (WHO, 2005b).

Os respondentes do grupo espera apresentaram pior avaliação da faceta do WHOQOL-OLD, atividades passadas, presentes e futuras, que descreve a satisfação sobre conquistas e anseios na vida. Constata-se na lista de espera para residir em ILPI, pessoas idosas com desejo de melhorar as condições de vida, ter garantido cuidados futuros, conforto, segurança, tranquilidade e poder construir novas relações sociais.

Também se ouviram a própria pessoa idosa, familiares e não familiares revoltados com a morosidade na obtenção da vaga e inexistência de alternativas para o cuidado da pessoa idosa com limitações. Constatou-se ainda o constrangedor e lamentável percentual de $23 \%$ (78 pessoas) de óbitos de pessoas idosas que estavam em lista de espera aguardando ajuda. Dados do Sistema de Informações sobre Mortalidade do DATASUS (BRASIL, 2014) demonstram que nos últimos três anos de registro faleceram 7.917 pessoas idosas na cidade de João Pessoa, o que representa $11 \%$ da população média no período, dessa forma é possível dizer que a mortalidade no grupo espera foi duas vezes maior que o previsto.

As pessoas idosas dos grupos residente e não espera apresentaram médias baixas para os domínios meio ambiente e físico, sendo que as médias dos respondentes residente foram mais baixas, principalmente no domínio físico. As pesquisas sobre qualidade de vida com pessoas idosas não institucionalizadas corroboram os dados referentes ao não espera (PASKULIN, 2006; BRAGA et al., 2011; PEREIRA et al., 2006) assim como aos achados das pesquisas com pessoas idosas institucionalizadas (ALMEIDA; RODRIGUES, 2008) que referem maior comprometimento da capacidade funcional em relação ao grupo social não institucionalizado.

Para Braga et al. (2011), em estudo realizado com pessoas idosas residentes em Juiz de Fora/MG, as médias mais baixas de avaliação ocorreram no domínio meio ambiente. A suposta justificativa para o ocorrido está nas precárias oportunidades de lazer ofertadas pela comunidade e na inexistência em localidades próximas. A pessoa idosa não disponibiliza de incentivos para buscar em outras regiões e as dificuldades são inúmeras: baixa renda, problema com transporte, violência, falta de companhia, entre outras.

Na pesquisa realizada por Dias, Carvalho e Araújo (2013), também ocorreu uma percentagem baixa na avaliação do domínio meio ambiente em relação aos demais domínios. Ela faz referência à importância de um ambiente que 
a pessoa idosa possa andar com segurança nas calçadas, ir ao parque ou até mesmo tomar um transporte público, estimula o exercício da atividade e mantém o idoso socialmente ativo.

As facetas do instrumento WHOQOL-OLD, que obtiveram as menores médias nos grupos residente e não espera, foram autonomia e atividades passadas, presentes e futuras. Achados semelhantes foram apresentados por pesquisadores que avaliaram a qualidade de vida da pessoa idosa institucionalizada (NUNES; MENEZES; ALCHIERI, 2010) não institucionalizada (BELTRAME, 2008; SERBIM; FIGUEIREDO, 2011) e na comparação das duas populações (RAMOS, 2008). Essa faceta sofre considerada influência da avaliação do domínio meio ambiente, pois quando inseguros limitam as atividades sociais do idoso, passam a sair menos sozinhos, principalmente os não institucionalizados, além de favorecer isolamento e consequentemente alterações psíquicas, podendo refletir em sua autonomia (DIAS; CARVALHO; ARAÚJO, 2013). Segundo Neri (2010) um dos grandes desafios no aumento da expectativa de vida é a preservação da qualidade de vida nas presenças das ameaças de restrição da autonomia e da independência, sendo proporcionadas pelas mudanças no estado de saúde e também pelo empobrecimento da vida social.

\section{Considerações finais}

Ficou evidente, tanto no instrumento genérico, quanto no específico para a pessoa idosa, a diferença que existe na avaliação da qualidade de vida entre os três grupos. Entretanto, os escores do residente foram mais próximos ao do não espera, enquanto que os produzidos pelo grupo espera foram consideravelmente inferiores. Portanto, conclui-se que a institucionalização não proporciona piora na qualidade de vida da pessoa idosa. A percepção dessa qualidade já está comprometida quando se procura ILPI. Sendo assim, a ILPI, que para muitos estudos representa um "inferno", para as pessoas idosas em lista de espera representa uma "salvação".

É de aquiescência a ideia de que, para a pessoa idosa, o lugar ideal seria estar em sua residência, tendo o suporte familiar, desde que tenha reflexo positivo em sua qualidade de vida. As famílias brasileiras enfrentam muitos obstáculos em relação ao cuidar da pessoa idosa com comprometimento cognitivo e/ou funcional em domicílio. Existe carência de programas que auxiliem na orientação e acompanhamento do cuidado domiciliar; que forneçam apoio de cuidadores formais adequadamente capacitados sem comprometer o orçamento familiar; que possam disponibilizar de equipe multidisciplinar para 
acompanhar em domicílio a pessoa idosa incapacitada. Os projetos devem incluir ações preventivas e resolutivas, considerando a heterogeneidade desta população. Considera-se importante que os gestores públicos e a sociedade em geral conheçam a realidade das pessoas idosas em lista de espera e possam buscar intervenções que proporcionem melhoria da qualidade de vida desse contingente populacional.

\author{
QUALITY OF LIFE OF ELDERLY PEOPLE \\ AS LEVEL OF INSTITUTIONALIZATION
}

abstract

Introduction: Studies shows the negative effect of the elderly's institutionalization on quality of life. Objective: To evaluate possible differences in the elderly's quality of life on waiting lists for living in long-term institutions (waiting list), living in institution (resident) and that not in the waiting list (control). Method: Cross-sectional study. Fifty respondents in each group were paired by sex, age and education level. WHOQOL-BREF and WHOQOL-OLD instruments were used. Results: There was significant difference in all domains and issues of the WHOQOL-BREF among the three groups, with the mean total scores of: $62.0 \pm 10.61,44.1 \pm 13.63$ and $68.8 \pm 7.07$, respectively for the resident, waitlisted and control groups. Important differences were also identified in the evaluation of the WHOQOL-OLD. The total score for the resident was $60.4 \pm 9.88$, waitlisted: $48.5 \pm 12.15$ and control: $68.5 \pm 7.90$ ( $p<0.0001$ ). Conclusion: Institutionalization does not worsen the elderly's quality of life; the perception of this quality can already be compromised when seeking institutionalization.

keywords

Aging. Elder. Quality of Life. Waiting lists.

referências

ALMEIDA, Antônio José Pereira dos Santos; RODRIGUES, Vitor Manuel Costa Pereira. A qualidade de vida da pessoa idosa institucionalizada em lares. Revista Latino-Americana de Enfermagem, Ribeirão Preto, v. 16, n. 6, p. 1025-1031, 2008.

BELTRAME, Vilma. Qualidade de vida de idosos diabéticos. 2008. 101 f. Tese (Doutorado em Gerontologia Biomédica)-Pontifícia Universidade Católica, Porto Alegre, 2008. 
BRAGA, Maria Cecília Portugal et al. Qualidade de vida medida pelo WHOQOL-BREF: estudo com idosos residentes em Juiz de Fora. Revista Atenção Primária à Saúde, Juiz de Fora, v. 14, n. 1, p. 93-100, 2011

BRASIL. Sistema de Informações sobre Mortalidade. DATASUS. Disponível em: <http:// tabnet.datasus.gov.br/cgi/deftohtm.exe?sim/cnv/obt10uf.def>. Acesso em: 1 fev. 2014.

Ministério da Saúde. Envelhecimento e saúde da pessoa idosa. Brasília, DF: Ministério da Saúde, 2007. (Cadernos de Atenção Básica, 19) (Série A. Normas e Manuais Técnicos).

Política Nacional de Saúde da Pessoa Idosa. Brasília, DF, 2006. Disponível em: <http://bvsms.saude.gov.br/bvs/saudelegis/gm/2006/prt2528_19_10_2006.html>. Acesso em: 20 maio 2006

CAMARANO, Ana Amélia; KANSO, Solange. As instituições de longa permanência para idosos no Brasil. Revista Brasileira de Estudos de População, Rio de Janeiro, v. 27, n. 1, p. 232-235, 2010 a.

. Como as famílias brasileiras estão lidando com idosos que demandam cuidados e quais as perspectivas futuras? a visão mostrada pelas PNADS. In: CAMARANO, A. A. (Org.). Cuidados de longa duração para a população idosa: um novo risco social a ser assumido? Rio de Janeiro: IPEA, 2010b. p. 93-122.

CAMARANO, Ana Amélia; PASINATO, Maria Tereza. O envelhecimento populacional na agenda das políticas públicas. In: CAMARANO, A. A. (Org). Os novos idosos brasileiros: muito além dos 60? Rio de Janeiro: IPEA, 2004. p. 253-292.

CAMARANO, Ana Amélia; SCHARFSTEIN, Eloisa Adler. Instituição de longa permanência para idosos: abrigo ou retiro? In: CAMARANO, A. A. (Org.). Cuidados de longa duração para a população idosa: um novo risco social a ser assumido? Rio de Janeiro: IPEA, 2010. p. 163-186.

CHRISTOPHE, Micheline; CAMARANO, Ana Amélia. Dos asilos às instituições de longa permanência: uma história de mitos e preconceitos? In: CAMARANO, A. A. (Org.). Cuidados de longa duração para a população idosa: um novo risco social a ser assumido? Rio de Janeiro: IPEA, 2010. p. 145-162.

DANILOW, Milena Zamian et al. Perfil epidemiológico e psicossocial de idosos institucionalizados do Distrito Federal. Comunicação em Ciências da Saúde, Brasília, DF, v. 18, n.1 p. 9-16, 2007.

DIAS, Daniela Silva Gonçalves; CARVALHO, Carolina da Silva; ARAÚJO, Cibelle Vanessa de. Comparação da percepção subjetiva de qualidade e bem-estar de idosos que vivem sozinhos, com a família e institucionalizados. Revista Brasileira de Geriatria e Gerontologia, Rio de Janeiro, v. 16, n. 1, p. 127-138, 2013.

FLECK, M. P. A. Problemas conceituais e qualidade de vida. In: FLECK, M. P. A. (Org.). A avaliação de qualidade de vida: guia para profissionais da saúde. Porto Alegre: Artmed, 2008. p. 19-28.

FLECK, M. P. A. et al. Aplicação da versão em português do instrumento abreviado de avaliação de qualidade de vida WHOQOL-BREF. Revista de Saúde Pública, São Paulo, v. 34, n. 2, p. 178-183, 2000.

FREITAS, Mariana Ayres Vilhena; SCHEICHER, Marcos Eduardo. Qualidade de vida de idosos institucionalizados. Revista Brasileira de Geriatria e Gerontologia, Rio de Janeiro, v. 13, n. 3, p. 395-402, 2010

FOLSTEIN, Marshal F:; FOLSTEIN, Suzan E:; MCHUGH, Paul R. Mini-mental State: a practical method for grading the connective state of patients for the clinician. Journal Psychiatric Research, v. 3, n. 12, p. 189-98, 1975.

LITIVOC, Júlio; BRITO, Francisco Carlos. Envelhecimento: prevenção e promoção da saúde. São Paulo: Atheneu, 2004. 
MINCATO, Paula Cristina; FREITAS, Cíntia La Rocha. Qualidade de vida dos idosos residentes em instituições asilares da cidade de Caxias do Sul-RS. Revista Brasileira de Ciências do Envelhecimento, Passo Fundo, v. 4, n. 1, p. 127-38, 2007.

NERI, Anita Liberalesso. Desafios ao bem-estar físico e psicológico enfrentados por idosos cuidadores no contexto da família: dados do FIBRA Campinas. In: CAMARANO, A. A. (Org.). Cuidados de longa duração para a população idosa: um novo risco social a ser assumido? Rio de Janeiro: IPEA, 2010. p. 303-36.

Palavras-chave em gerontologia. 3. ed. Campinas: Alínea, 2008.

Atitudes e preconceitos em relação à velhice. In:

(Org.). Idosos no

Brasil: vivências, desafios e expectativas na terceira idade. São Paulo: Fundação Perseu Abramo, 2007. p. 33-46.

NUNES, Vilane Medeiros de Araújo; MENEZES, Rejane Maria Paiva; ALCHIERI, José Carlos. Avaliação da qualidade de vida em idosos institucionalizados no município de Natal, Estado do Rio Grande do Norte. Acta Scientiarum: health sciences, Maringá v. 32, n. 2, p. 119-26, 2010 .

OLIVEIRA, Daiane Aparecida et al. Estudo Comparativo da Qualidade de Vida de idosos em cuidado domiciliar. Revista Faculdade Montes Belos, v. 8, n. 3, p. 142-179, 2015.

OLIVEIRA, Elizabety Regina Araújo; GOMES, Maria José; PAIVA, Karina Mary. Institucionalização e qualidade de vida de idosos da região metropolitana de Vitória-ES. Escola Anna Nery, Rio de Janeiro, v. 15, n. 3, p. 518-23, 2011.

OLIVEIRA, Mirna Poliana Furtado; NOVAES, Maria Rita Carvalho Garbi. Perfil socioeconômico, epidemiológico e farmacoterapêutico de idosos institucionalizados de Brasília, Brasil. Ciências \& Saúde Coletiva, Rio de Janeiro, v. 18, n. 4, p. 1069-78, 2013

PASKULIN, Lisiane Manganelli Girardi. Fatores associados à qualidade de vida de idosos de um distrito sanitário de Porto Alegre/RS. 2006. 165 f. Tese (Doutorado em Ciências)-Universidade Federal de São Paulo, São Paulo, 2006.

PEREIRA, Renata Junqueira et al. Contribuição dos domínios, físico, social, psicológico e ambiental para a qualidade de vida global de idosos. Revista de Psiquiatria do Rio Grande do Sul, Porto Alegre, v. 28, n. 1, p. 27-38, 2006.

POLARO, Sandra Helena Isse et al. Idosos residentes em instituições de longa permanência para idosos da região metropolitana de Belém-PA. Revista Brasileira de Geriatria e Gerontologia, Rio de Janeiro, v. 15, n. 4, p. 777-84, 2012.

POLLO, Sandra Helena Lima; ASSIS, Mônica. Instituições de longa permanência para idosos - ILPI's: desafios e alternativas no município do Rio de Janeiro. Revista Brasileira de Geriatria e Gerontologia, Rio de Janeiro, v. 11, n. 1, p. 29-43, 2008.

POWER, Mick. Qualidade de vida visão geral do projeto WHOQOL. In: FLECK, Marcelo Pio de Almeida et al. (Org.). A avaliação de qualidade de vida: guia para profissionais da saúde. Porto Alegre: Artmed, 2008. p. 48-59.

QUEIROZ, Gleicimara Araújo. Qualidade de vida em Instituições de Longa permanência para idosos: considerações a partir de um modelo alternativo de assistência. 2010. 140 f. Dissertação (Mestrado em Psicologia)-Universidade Federal de São João del-Rei, São João del-Rei, 2010.

RAMOS, Luciana Junqueira. Avaliação do estado nutricional, de seis domínios da qualidade de vida e da capacidade de tomar decisão de idosos institucionalizados e não-institucionalizados no municipio de Porto Alegre, RS. 2008. 68 f. Dissertação (Mestrado em Gerontologia Biomédica)-Pontifícia Universidade Católica, Porto Alegre, 2008.

SAGALLA, Raieli et al. Perfil antropométrico e qualidade de vida de idosos independentes institucionalizados e não institucionalizados, no município de Erechim, RS. Perspectiva, Florianópolis, n. 137, p. 81-92, 2013. 
SANTOS, Ariana Oliveira et al. Qualidade de vida de idosos residentes em Instituições de Longa Permanência: uma revisão sistemática. Revista Enfermagem Contemporânea, Salvador, v. 6, n. 2, p. 110-121, 2017

SERBIM, Andreivna Kharenine; FIGUEIREDO, Ana Elizabety Prado Lima. Qualidade de vida de idosos em um grupo de convivência. Scientia Médica, Porto Alegre, v. 21, n. 4, p. 166-72, 2011.

WORLD HEALTH ORGANIZATION (WHO). Versão final do instrumento WHOQOL e o manual de diretrizes para aplicação, 2005a. Disponível em: <https://www.ufrgs.br/ qualidep/qualidade-de-vida/projeto-whogol-old/manual>. Acesso em: 1 jan. 2012.

Envelhecimento ativo: uma política de saúde. Tradução de Suzana Gontijo. Brasília, DF: Organização Pan-Americana de Saúde, 2005b.

Data de submissão: 22/11/2015

Data de aprovação: 28/01/2018 